\title{
Analysis of the Effect of Entrepreneurial Spirit, Entrepreneurial Resources, Entrepreneurial Orientation, Community Culture to Entrepreneurial Performance with Work Motivation as Mediating Variables on Micro, Small and Medium Enterprise in East Java, Indonesia
}

\author{
H.Teman Koesmono \\ Lecturer of Business Faculty Widya Mandala Catholic University Surabaya \\ Surabaya, East Java-Indonesia
}

\begin{abstract}
This study aims to determine the extent to which Entrepreneurial performance can be influenced by variables directly or indirectly related, the research topic is Analysis of the influence of Entrepreneurial Spirit, Entrepreneurial Resources, Entrepreneurial Orientation, Community Culture on Entrepreneurial Performance with mediating variables Work motivation on MSME Province of East Java. The results showed that the entrepreneurial spirit, entrepreneurial resources, entrepreneurial orientation, community culture significantly influence entrepreneurial motivation and entrepreneurial resources, entrepreneurial orientation, significantly influence entrepreneurial performance. In addition, the results of this study prove that the entrepreneurial spirit and community culture have no effect on entrepreneurial performance. This research is useful for entrepreneurs in their respective regions, especially in East Java Province and to pay attention to the variables that can affect their performance with the hope that the business can be successful in accordance with its expectations.
\end{abstract}

Keywords: entrepreneurial spirit, entrepreneurial resources, entrepreneurial orientation, community culture, work motivation and entrepreneurial performance

DOI: $10.7176 / \mathrm{JCSD} / 52-06$

Publication date:October $31^{\text {st }} 2019$

\section{Background}

Economic activity and growth of a region contributes to national-level economic growth, with each province in Indonesia competing with one another to improve economic growth by utilizing the existence of its geographical area. The geographical area of an area will determine the productivity of the economic activities of the area concerned, by utilizing its natural resources whether it is the area of agriculture (agriculture), the area of trade (trading) or whether the area of manufacture (industry). To manage these economic activities requires reliable economic actors both at the level of micro, small and middle business fields respectively. East Java is a province that has a large population of MSME activities (Micro, Small and Medium Enterprises). Especially regarding the condition of MSMEs (Micro, Small and Medium Enterprises) in East Java, it is very encouraging because it contributes to significant economic growth, this is inseparable from the ability of MSME entrepreneurs who can diligently develop their businesses through creative and innovative activities. The contribution of MSME to the economy of East Java (East Java) experienced a significant increase, in 2012 it contributed to the GRDP of 54.98 percent and in 2016 amounted to 57.62 percent. Besides that the realization of investment from MSMEs in 2017 reached Rp.152.98 trillion and the first semester of 2018 amounted to 95.95 trillion, an increase of 22.87 percent from the same period in 2017. The perspective of the development or growth of MSMEs also shows encouraging things, namely in 2012 it reached 6.8 million and in 2017 it reached 9.59 million. (4.61 million in the nonagricultural sector and 4.98 million in the agricultural sector). This contributed to other forms of decreasing unemployment and poverty, MSMEs absorbed workforce 18.95 million out of the 20.16 million, then more than 90 percent of the workforce was absorbed by MSMEs (Dinkop and MSMEs of East Java Province). In connection with these data, of course, any variables that can encourage the growth of entrepreneurs include 1) Other entrepreneurial spirits owned by individuals as entrepreneurs 2) Entrepreneurial resources, 3) Entrepreneurial Orientation 4) Culture of the business environment, these four variables will affect the work motivation of entrepreneurs and their performance. Previous studies have been conducted by: Widyatnoto (2013) showed that the spirit of entrepreneurship and family culture influences motivation. Purwanto and Trihudiyatmanto (2018) showed that the entrepreneurial orientation influences business performance. Aramand (2013) showed that the culture influence to entrepreneurial motivation. Sumantri (2013) showed that the The Spirit of Entrepreneurship influences the Performance of Entrepreneurs in the Home Food Industry. Fornoni et al (2012), showed that the Resource of Entrepreneurship influences Performance of Entrepreneurs. Fauni (2017) conducted a research that the result is that entrepreneurial orientation influences business performance. Adri (2013) showed that the Culture of the work environment influences MSMEs. Gemina et al (2016) showed that the Business Motivation influences 
the Capability of Small and Medium Enterprises in Snack Food Industry. Machmud and Sidharta (2016) showed that the Entrepreneurial Motivation Influence to Business Performance of SMEs. Based on the background of the condition of MSMEs in East Java and the results of previous studies on variables that can affect Entrepreneurial Performance, it is necessary to conduct research entitled The Effect of Entrepreneurial Soul, Entrepreneurial Resources, Entrepreneurial Orientation and Community Culture on Entrepreneurial Performance with mediation variables Work Motivation in MSMEs (Micro, Small and Medium Enterprises) in East Java.

\section{Theoritical Review}

Being an entrepreneur requires reliable supplies, meaning that the basic capital owned must be strong because the challenges of being entrepreneurs are very high in the future, and not infrequently becoming an entrepreneur but failing halfway. In the business world, there is a condition of uncertainty, which is an uncertain condition in managing a business in any field. That is because the climate and business environment always changes depending on the economic and political conditions of a country or its people. Sometimes the policy of the government towards business always changes or adjusted to the existing regulations on trade activities in the community such as the imposition of tax rates for special goods that have high export and import values and others. An entrepreneur before implementing his business ideas certainly requires a strong entrepreneurial spirit so that what is planned can be in accordance with his expectations. Kasali et al (2010: 12) The spirit of entrepreneurship is the spirit of independence to look for sources of income or income by opening your own business and by channeling creative and innovative ideas so as to produce more value from an item. In managing MSME, of course, it requires adequate resources in accordance with the line of business occupied by an entrepreneur. Business resources can be in the form of capital, material, place of business, labor and business licensing from the government which is a strategic location for the business. According to Subanar (2012:150), entrepreneurial resources are resources that are able to combine the interests of natural resources, human resources and capital resources as a requirement for running a business. Entrepreneurial resources are very strategic in planning to build MSMEs in any region, because business resources are components that will be processed into finished goods, the availability must be sufficient from time to time. According to Suryana (2013: 80), entrepreneurial orientation is the direction of one's activities based on capital, willingness, ability and knowledge as well as skills regarding entrepreneurship that is carried out. The existence of the culture of the community around the MSME industrial area also contributes to the growth of entrepreneurs, because the community environment is a mirror of social life in the area concerned. For example, agricultural communities certainly support agriculture will be stronger to give color to people's lives, so will happen to people who know more about business will be affected by the existing business climate and so on. The existence of the community in one particular area can describe the behavior patterns of the surrounding community and represent the existing community and can be said to be a member of the organization in that community. Considering that a group of people can describe an organization in that community, it will certainly have a culture of life as a reference for behavior. According to Robbin and Judge (2015: 254), culture is a system of shared meanings shared by its members and distinguishes it from other members of different organizations. In the environment in an entrepreneurial area, an entrepreneurial culture will be found, especially in the environment or location of the MSME. Entrepreneurial culture is the values that are believed to be true in an effort to build an entrepreneurial spirit and entrepreneurial motivation to continue to develop, for examples of animal skin craftsmen in Tanggulangin are certain areas where the majority of people are entrepreneurs making products from animal skins for the needs of the wider community such as shoes, bags and other things. It can be said that the community has a culture of entrepreneurial spirit, especially at the MSME level in processing animal skins for domestic use. According to Saiman (2014: 25), motivation to become an entrepreneur usually appears by itself, after having enough stock to manage the business and be mentally prepared totally. We already know and understand that motivation is an impulse from a person both comes from himself or from outside to meet his needs and desires so that the continuity of his life can be guaranteed. According to Nitisusastro (2009: 118), motivation is a process that refers to causes that cause a person to behave. Likewise for an entrepreneur, he certainly wants his business to run in accordance with his expectations and requires factors that can strengthen his motivation for entrepreneurship. Entrepreneurial performance requires strong businesses and individual character as well as a strong entrepreneurial culture both from themselves and from their environment. In order to be achieved in accordance with the issued capital (business capital can develop in accordance with the performance achieved). As an entrepreneur, besides running his business, he also doubles as a leader in an organization, therefore the success of an entrepreneur will be influenced by his leadership towards himself and others. According to Nourthouse (2013: 5) Leadership is a process in which individuals influence a group of individuals to achieve common goals. Understanding is a process is nothing but running a transaction to influence followers and be influenced by followers in their business activities. To support the performance achievement of a business carried out by entrepreneurs, it requires efforts to identify several behavioral factors such as entrepreneurial spirit, entrepreneurial resources, entrepreneurial orientation, community culture besides the extent of work motivation and leadership to strengthen motivation so that performance can be achieved maximum. MSMEs need special 
attention from the government so that their existence can still contribute to economic growth in the areas where MSMEs are located. The attention can be in the form of financial or non-financial. Specifically for finance, it can be in the form of soft loans from government financial institutions. Non-financial matters can be in the form of assistance or guidance so that the business can continue and its products can compete at the national and international level. In addition, there are businesses that always foster creativity and innovation, continue to be encouraged in order to create products that encourage the growth of the creative economy.

\section{Research Conceptual Framework}

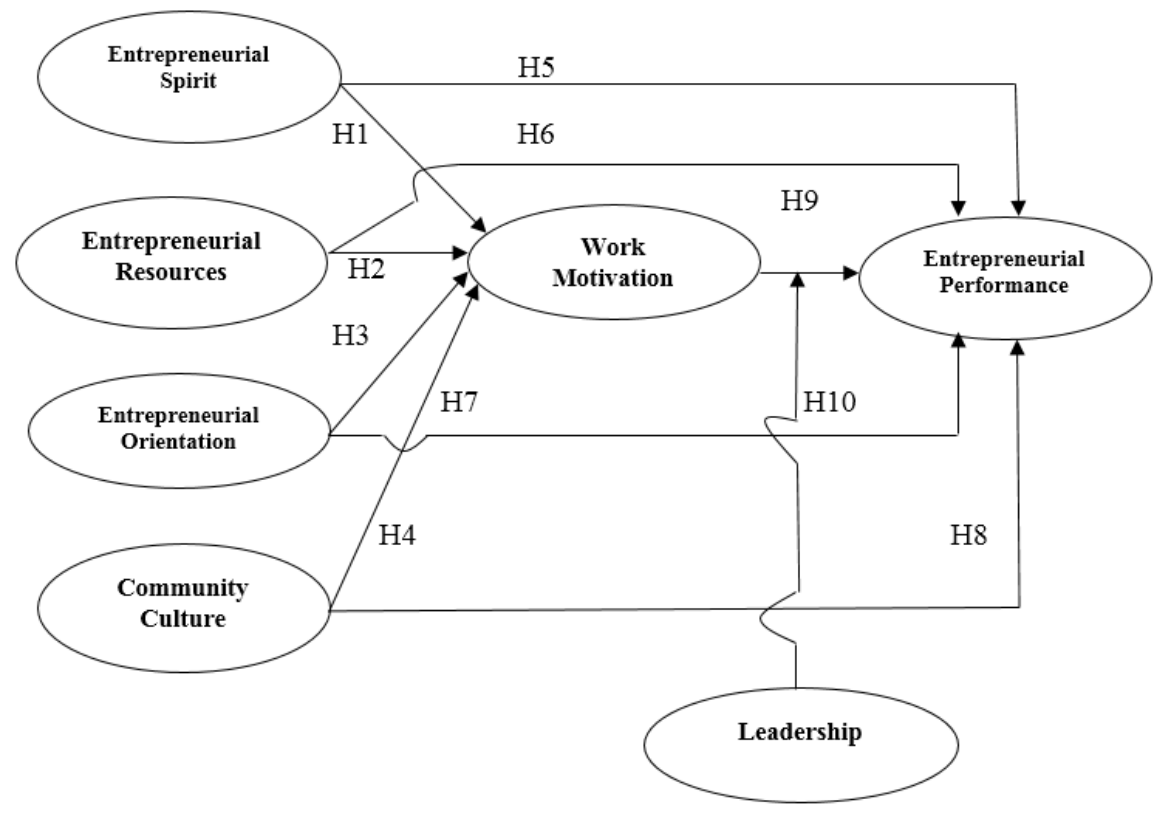

\section{Hypothesis}

Hypothesis on this research are as follow:

1. Entrepreneurial spirit influences work motivation

2. Entrepreneurial Resources affect Work Motivation

3. Entrepreneurial Orientation has a negative effect on Work Motivation

4. Community culture influences work motivation

5. Entrepreneurial spirit influences entrepreneurial performance

6. Entrepreneurial Resources influence Entrepreneurial Performance

7. Entrepreneurial Orientation influences Entrepreneurial Performance

8. Community Culture influences Entrepreneurial Performance

9. Work motivation has an effect on Entrepreneurial Performance

10. Leadership is a moderating variable of influence between Work Motivation on Entrepreneurial Performance

\section{Research methods}

\subsection{Research design}

Based on the background of the problem it can be classified that this study uses a hypothesis. This research is based on the facts or current conditions of MSMEs in East Java. This study presents the extent to which Entrepreneurial Resources in Entrepreneurship, Entrepreneurial Orientation and Community Culture affect Work Motivation and Entrepreneurial Performance with Leadership as Moderate Variables in MSMEs (Micro, Small and Medium Enterprises) ) in East Java.

\subsection{Data types and sources}

The type of data is the interval scale (Likert scale) shown with the same distance between one number from another starting from the smallest to the largest number which is 1 (strongly disagree), 2 (disagree), 3 (neutral), 4 (agree) and 5 (totally agree). While the source of the data used is primary by distributing questionnaires to respondents, namely the MSMEs in East Java. 
5.3 The technique of taking and the number of samples

The sampling technique used was random sampling from the population of SMEs in East Java Sugiyono (2010: 91) The number of samples used was 100-200 people. The number of indicators is 35 so that the number of samples using $35 \times 6=195$ people

\subsection{Technical analysis of data}

The data analysis technique will be used using SEM (Structural Equation Modeling) with the AMOS 4.0 Program. Ferdinand (2014: 110)

\subsection{Regression Equation}

$\mathrm{Y} 1=\mathrm{aX} 1+\mathrm{bX} 2+\mathrm{cX} 3+\mathrm{dX} 4$

$\mathrm{Y} 2=\mathrm{eX} 1+\mathrm{fX} 2+\mathrm{gX} 3+\mathrm{hX} 4+\mathrm{iY} 1$

$\mathrm{Z}=$ Moderate variable

\subsection{Variables Identification}

1.Independent variables: Entrepreneurial Spirit, Entrepreneurial Resources, Entrepreneurial Orientation and Community Culture (X1,X2,X3.X4)

2.Dependent variables: Work Motivation, Entrepreneurial Performance (Y1,Y2)

3. Moderate variables: Leadership $(Z)$

\subsection{Definition of Variable Operations}

1. Entrepreneurial Spirit is the spirit of someone's independence towards the fulfillment of their needs through productive efforts to generate income.

2. Entrepreneurial Resources are various business resources that are able to combine the interests of entrepreneurs in producing goods or services

3. Entrepreneurial Orientation is the direction of a person's activities based on capital and enthusiasm to increase the value of goods to be more useful.

4. Community Culture is the values that exist in the community that can encourage productive behavior and benefit their lives

5. Motivation Work work is encouragement that comes from inside and outside so that someone does an activity to meet their wants and needs

6. Entrepreneurial Performance is the result of entrepreneurial activities within a certain period that can be measured quantitatively and qualitatively

7. Leadership is a pattern of activity of a leader in managing his business and subordinates or followers.

\section{Research Result}

6.1 Descriptive Statistics

\begin{tabular}{|c|c|c|c|c|c|c|c|c|}
\hline \multirow{2}{*}{ Variables } & \multirow{2}{*}{ Indicators } & \multicolumn{5}{|c|}{ Answer Percentage } & \multirow{2}{*}{$\begin{array}{c}\text { Mean } \\
\text { Indicator }\end{array}$} & \multirow{2}{*}{$\begin{array}{c}\text { Mean } \\
\text { Variable }\end{array}$} \\
\hline & & STS & TS & $\mathrm{N}$ & $\mathrm{S}$ & SS & & \\
\hline \multirow{5}{*}{$\begin{array}{c}\text { Entrepreneurial } \\
\text { Spirit (X1) }\end{array}$} & $\mathrm{X} 1.1$ & 0,0 & 0,0 & 15,5 & 51,5 & 33,0 & 4,18 & \multirow{5}{*}{4,11} \\
\hline & $\mathrm{X} 1.2$ & 0,0 & 0,0 & 14,0 & 61,5 & 24,5 & 4,11 & \\
\hline & $\mathrm{X} 1.3$ & 0,0 & 0,0 & 30,5 & 50,0 & 19,5 & 3,89 & \\
\hline & X1.4 & 0,0 & 0,0 & 8,5 & 51,0 & 40,5 & 4,32 & \\
\hline & $\mathrm{X} 1.5$ & 0,0 & 0,0 & 20,5 & 53,5 & 26,0 & 4,06 & \\
\hline \multirow{5}{*}{$\begin{array}{l}\text { Entrepreneurial } \\
\text { Resources (X2) }\end{array}$} & $\mathrm{X} 2.1$ & 0,0 & 0,0 & 18,0 & 47,5 & 34,5 & 4,17 & \multirow{5}{*}{4,12} \\
\hline & $\mathrm{X} 2.2$ & 0,0 & 0,0 & 14,5 & 57,0 & 28,5 & 4,14 & \\
\hline & $\mathrm{X} 2.3$ & 0,0 & 0,0 & 19,5 & 45,5 & 35,0 & 4,16 & \\
\hline & $\mathrm{X} 2.4$ & 0,0 & 0,0 & 23,0 & 47,5 & 29,5 & 4,07 & \\
\hline & $\mathrm{X} 2.5$ & 0,0 & 0,0 & 23,0 & 48,5 & 28,5 & 4,06 & \\
\hline \multirow{3}{*}{$\begin{array}{c}\text { Entrepreneurial } \\
\text { Orientation (X3) }\end{array}$} & X3.1 & 0,0 & 10,0 & 25,0 & 47,0 & 18,0 & 3,73 & \multirow{3}{*}{4,05} \\
\hline & X3.2 & 0,0 & 0,5 & 11,0 & 47,5 & 41,0 & 4,29 & \\
\hline & $\mathrm{X} 3.3$ & 0,0 & 0,0 & 26,5 & 54,5 & 19,0 & 3,93 & \\
\hline
\end{tabular}




\begin{tabular}{|c|c|c|c|c|c|c|c|c|}
\hline \multirow{4}{*}{ Variables } & \multirow{3}{*}{$\begin{array}{c}\text { Indicators } \\
\text { X3.4 } \\
\end{array}$} & \multicolumn{5}{|c|}{ Answer Percentage } & \multirow{2}{*}{$\begin{array}{l}\text { Mean } \\
\text { Indicator }\end{array}$} & \multirow{4}{*}{$\begin{array}{c}\text { Mean } \\
\text { Variable }\end{array}$} \\
\hline & & STS & TS & $\mathrm{N}$ & $\mathrm{S}$ & SS & & \\
\hline & & 0,0 & 0,0 & 22,5 & 48,5 & 29,0 & 4,07 & \\
\hline & X3.5 & 0,0 & 1,0 & 11,0 & 49,0 & 39,0 & 4,26 & \\
\hline \multirow{5}{*}{$\begin{array}{l}\text { Community } \\
\text { Culture (X4) }\end{array}$} & $\mathrm{X} 4.1$ & 0,0 & 0,0 & 13,0 & 43,0 & 44,0 & 4,31 & \multirow{5}{*}{4,01} \\
\hline & $\mathrm{X} 4.2$ & 0,0 & 0,0 & 30,0 & 49,5 & 20,5 & 3,91 & \\
\hline & $\mathrm{X} 4.3$ & 0,0 & 0,0 & 31,5 & 48,0 & 20,5 & 3,89 & \\
\hline & $\mathrm{X} 4.4$ & 0,0 & 0,0 & 23,5 & 52,0 & 24,5 & 4,01 & \\
\hline & $\mathrm{X} 4.5$ & 0,0 & 0,0 & 28,5 & 48,5 & 23,0 & 3,95 & \\
\hline \multirow{5}{*}{$\begin{array}{c}\text { Work } \\
\text { Motivation (Y1) }\end{array}$} & Y1.1 & 0,0 & 0,0 & 25,0 & 51,5 & 23,5 & 3,99 & \multirow{5}{*}{4,13} \\
\hline & $\mathrm{Y} 1.2$ & 0,0 & 6,0 & 32,5 & 36,0 & 25,5 & 3,81 & \\
\hline & $\mathrm{Y} 1.3$ & 0,0 & 0,0 & 9,5 & 55,5 & 35,0 & 4,26 & \\
\hline & Y1.4 & 0,0 & 0,0 & 9,0 & 54,5 & 36,5 & 4,28 & \\
\hline & Y1.5 & 0,0 & 0,0 & 8,5 & 52,0 & 39,5 & 4,31 & \\
\hline \multirow{5}{*}{$\begin{array}{l}\text { Entrepreneurial } \\
\text { Leadership (Z) }\end{array}$} & $\mathrm{Z} 1.1$ & 0,0 & 0,0 & 4,5 & 48,0 & 47,5 & 4,43 & \multirow{5}{*}{4,24} \\
\hline & $\mathrm{Z} 1.2$ & 0,0 & 0,0 & 11,0 & 56,0 & 33,0 & 4,22 & \\
\hline & $\mathrm{Z1} 1.3$ & 0,0 & 0,0 & 11,5 & 49,0 & 39,5 & 4,28 & \\
\hline & $\mathrm{Z} 1.4$ & 0,0 & 0,0 & 22,0 & 57,0 & 21,0 & 3,99 & \\
\hline & $\mathrm{Z} 1.5$ & 0,0 & 0,0 & 12,0 & 48,0 & 40,0 & 4,28 & \\
\hline \multirow{5}{*}{$\begin{array}{l}\text { Entrepreneurial } \\
\text { Performance } \\
\text { (Y2) }\end{array}$} & Y 2.1 & 0,0 & 0,0 & 31,0 & 48,0 & 21,0 & 3,90 & \multirow{5}{*}{4,09} \\
\hline & $\mathrm{Y} 2.2$ & 0,0 & 0,0 & 12,0 & 45,5 & 42,5 & 4,31 & \\
\hline & Y2.3 & 0,0 & 0,0 & 23,5 & 51,5 & 25,0 & 4,02 & \\
\hline & Y2.4 & 0,0 & 0,0 & 26,5 & 48,5 & 25,0 & 3,99 & \\
\hline & $\mathrm{Y} 2.5$ & 0,0 & 0,0 & 13,0 & 49,0 & 38,0 & 4,25 & \\
\hline
\end{tabular}

\subsection{CFA test} CFA Exogen Construct

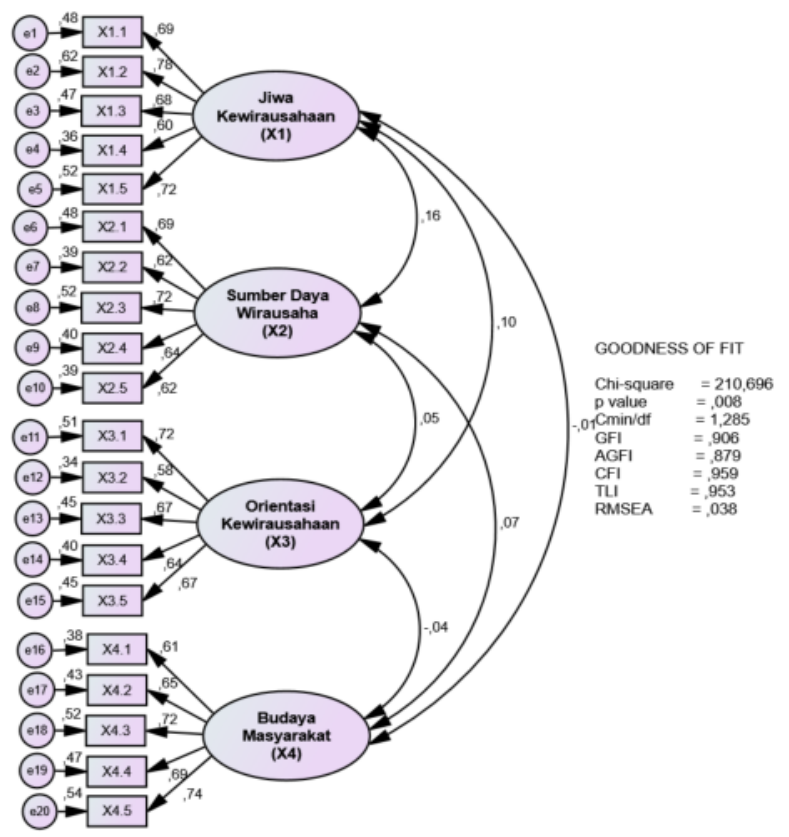




\begin{tabular}{|c|c|c|c|c|}
\hline Variables & Indicator & Factor Loading & Req & Explanation \\
\hline \multirow{5}{*}{$\begin{array}{l}\text { Entrepreneurial } \\
\text { Spirit (X1) }\end{array}$} & X1.1 & 0,694 & $\geq 0,50$ & Valid \\
\hline & $\mathrm{X} 1.2$ & 0,785 & $\geq 0,50$ & Valid \\
\hline & $\mathrm{X} 1.3$ & 0,683 & $\geq 0,50$ & Valid \\
\hline & $\mathrm{X} 1.4$ & 0,598 & $\geq 0,50$ & Valid \\
\hline & $\mathrm{X} 1.5$ & 0,723 & $\geq 0,50$ & Valid \\
\hline \multirow{5}{*}{$\begin{array}{l}\text { Entrepreneurial } \\
\text { Resources (X2) }\end{array}$} & $\mathrm{X} 2.1$ & 0,693 & $\geq 0,50$ & Valid \\
\hline & $\mathrm{X} 2.2$ & 0,625 & $\geq 0,50$ & Valid \\
\hline & $\mathrm{X} 2.3$ & 0,721 & $\geq 0,50$ & Valid \\
\hline & $\mathrm{X} 2.4$ & 0,636 & $\geq 0,50$ & Valid \\
\hline & $\mathrm{X} 2.5$ & 0,624 & $\geq 0,50$ & Valid \\
\hline \multirow{5}{*}{$\begin{array}{l}\text { Entrepreneurial } \\
\text { Orientation (X3) }\end{array}$} & $\mathrm{X} 3.1$ & 0,716 & $\geq 0,50$ & Valid \\
\hline & X3.2 & 0,584 & $\geq 0,50$ & Valid \\
\hline & $\mathrm{X} 3.3$ & 0,671 & $\geq 0,50$ & Valid \\
\hline & X3.4 & 0,635 & $\geq 0,50$ & Valid \\
\hline & X3.5 & 0,670 & $\geq 0,50$ & Valid \\
\hline \multirow{5}{*}{$\begin{array}{l}\text { Community } \\
\text { Culture (X4) }\end{array}$} & $\mathrm{X} 4.1$ & 0,614 & $\geq 0,50$ & Valid \\
\hline & $\mathrm{X} 4.2$ & 0,654 & $\geq 0,50$ & Valid \\
\hline & $\mathrm{X} 4.3$ & 0,720 & $\geq 0,50$ & Valid \\
\hline & $\mathrm{X} 4.4$ & 0,687 & $\geq 0,50$ & Valid \\
\hline & $\mathrm{X} 4.5$ & 0,738 & $\geq 0,50$ & Valid \\
\hline
\end{tabular}

\section{CFA Endogen Construct}

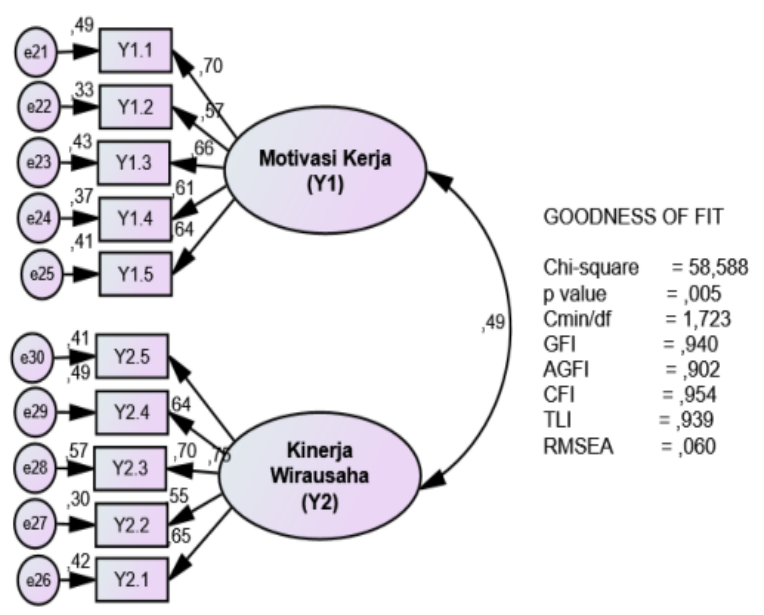

\begin{tabular}{|c|c|c|c|c|}
\hline Variables & Indicator & Factor Loading & Req & Explanation \\
\hline \multirow{4}{*}{$\begin{array}{c}\text { Work } \\
\text { Motivation (Y1) }\end{array}$} & Y1.1 & 0,699 & $\geq 0,50$ & Valid \\
\cline { 2 - 5 } & Y1.2 & 0,574 & $\geq 0,50$ & Valid \\
\cline { 2 - 5 } & Y1.3 & 0,659 & $\geq 0,50$ & Valid \\
\cline { 2 - 5 } & Y1.4 & 0,606 & $\geq 0,50$ & Valid \\
\cline { 2 - 5 } & Y1.5 & 0,639 & $\geq 0,50$ & Valid \\
\hline $\begin{array}{c}\text { Entrepreneurial } \\
\text { Performance }\end{array}$ & Y2.1 & 0,651 & $\geq 0,50$ & Valid \\
\cline { 2 - 5 } & Y2.2 & 0,551 & $\geq 0,50$ & Valid \\
\hline
\end{tabular}




\begin{tabular}{|c|c|c|c|c|}
\hline Variables & Indicator & Factor Loading & Req & Explanation \\
\hline \multirow{3}{*}{$($ Y2) } & Y2.3 & 0,753 & $\geq 0,50$ & Valid \\
\cline { 2 - 5 } & Y2.4 & 0,704 & $\geq 0,50$ & Valid \\
\cline { 2 - 5 } & Y2.5 & 0,639 & $\geq 0,50$ & Valid \\
\hline
\end{tabular}

CFA Moderating Construct

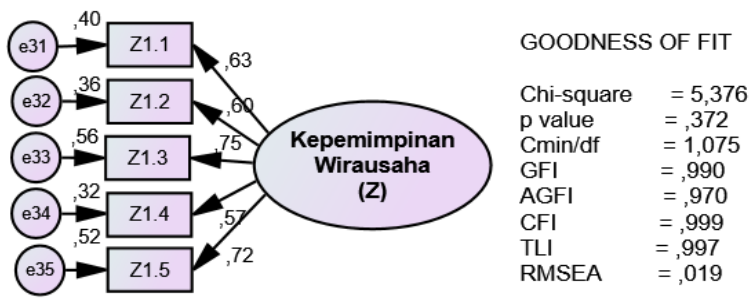

\begin{tabular}{|c|c|c|c|c|}
\hline Variables & Indicator & Factor Loading & Req & Explanation \\
\hline \multirow{4}{*}{$\begin{array}{c}\text { Entrepreneurial } \\
\text { Leadership (Z) }\end{array}$} & $\mathrm{Z} 1.1$ & 0,634 & $\geq 0,50$ & Valid \\
\cline { 2 - 5 } & $\mathrm{Z} 1.2$ & 0,604 & $\geq 0,50$ & Valid \\
\cline { 2 - 5 } & $\mathrm{Z} 1.3$ & 0,750 & $\geq 0,50$ & Valid \\
\cline { 2 - 5 } & $\mathrm{Z} 1.4$ & 0,565 & $\geq 0,50$ & Valid \\
\cline { 2 - 5 } & $\mathrm{Z} 1.5$ & 0,723 & $\geq 0,50$ & Valid \\
\hline
\end{tabular}

\subsection{Construct Reliability Test}

\begin{tabular}{|c|c|c|c|c|c|}
\hline Variables & Indicators & $\begin{array}{c}\text { Factor Loading } \\
(\mathrm{FL})\end{array}$ & $\mathrm{FL}^{2}$ & $\begin{array}{c}\text { Error } \\
\left(1-\mathrm{FL}^{2}\right)\end{array}$ & $\begin{array}{l}\text { Construct } \\
\text { Reliability }\end{array}$ \\
\hline \multirow{5}{*}{$\begin{array}{l}\text { Entrepreneurial } \\
\text { Spirit (X1) }\end{array}$} & $\mathrm{X} 1.1$ & 0,694 & 0,482 & 0,518 & \multirow{5}{*}{0,826} \\
\hline & $\mathrm{X} 1.2$ & 0,785 & 0,616 & 0,384 & \\
\hline & $\mathrm{X} 1.3$ & 0,683 & 0,466 & 0,534 & \\
\hline & X1.4 & 0,598 & 0,358 & 0,642 & \\
\hline & $\mathrm{X} 1.5$ & 0,723 & 0,523 & 0,477 & \\
\hline \multirow{5}{*}{$\begin{array}{l}\text { Entrepreneurial } \\
\text { Resources (X2) }\end{array}$} & $\mathrm{X} 2.1$ & 0,693 & 0,480 & 0,520 & \multirow{5}{*}{0,794} \\
\hline & $\mathrm{X} 2.2$ & 0,625 & 0,391 & 0,609 & \\
\hline & $\mathrm{X} 2.3$ & 0,721 & 0,520 & 0,480 & \\
\hline & X2.4 & 0,636 & 0,404 & 0,596 & \\
\hline & $\mathrm{X} 2.5$ & 0,624 & 0,389 & 0,611 & \\
\hline \multirow{5}{*}{$\begin{array}{c}\text { Entrepreneurial } \\
\text { Orientation (X3) }\end{array}$} & X3.1 & 0,716 & 0,513 & 0,487 & \multirow{5}{*}{0,791} \\
\hline & X3.2 & 0,584 & 0,341 & 0,659 & \\
\hline & $\mathrm{X} 3.3$ & 0,671 & 0,450 & 0,550 & \\
\hline & X3.4 & 0,635 & 0,403 & 0,597 & \\
\hline & $\mathrm{X} 3.5$ & 0,670 & 0,449 & 0,551 & \\
\hline \multirow{5}{*}{$\begin{array}{l}\text { Community } \\
\text { Culture (X4) }\end{array}$} & X4.1 & 0,614 & 0,377 & 0,623 & \multirow{5}{*}{0,814} \\
\hline & $\mathrm{X} 4.2$ & 0,654 & 0,428 & 0,572 & \\
\hline & $\mathrm{X} 4.3$ & 0,720 & 0,518 & 0,482 & \\
\hline & $\mathrm{X} 4.4$ & 0,687 & 0,472 & 0,528 & \\
\hline & $\mathrm{X} 4.5$ & 0,738 & 0,545 & 0,455 & \\
\hline Work & Y1.1 & 0,699 & 0,489 & 0,511 & 0,773 \\
\hline
\end{tabular}




\begin{tabular}{|c|c|c|c|c|c|}
\hline Variables & Indicators & $\begin{array}{l}\text { Factor Loading } \\
\text { (FL) }\end{array}$ & $\mathrm{FL}^{2}$ & $\begin{array}{c}\text { Error } \\
\left(1-\mathrm{FL}^{2}\right)\end{array}$ & $\begin{array}{l}\text { Construct } \\
\text { Reliability }\end{array}$ \\
\hline \multirow[t]{4}{*}{ Motivation (Y1) } & Y1.2 & 0,574 & 0,329 & 0,671 & \\
\hline & Y1.3 & 0,659 & 0,434 & 0,566 & \\
\hline & Y1.4 & 0,606 & 0,367 & 0,633 & \\
\hline & Y1.5 & 0,639 & 0,408 & 0,592 & \\
\hline \multirow{5}{*}{$\begin{array}{l}\text { Entrepreneurial } \\
\text { Performance } \\
\text { (Y2) }\end{array}$} & Y2.1 & 0,651 & 0,424 & 0,576 & \multirow{5}{*}{0,795} \\
\hline & Y2.2 & 0,551 & 0,304 & 0,696 & \\
\hline & Y2.3 & 0,753 & 0,567 & 0,433 & \\
\hline & Y2.4 & 0,704 & 0,496 & 0,504 & \\
\hline & Y2.5 & 0,639 & 0,408 & 0,592 & \\
\hline \multirow{5}{*}{$\begin{array}{l}\text { Entrepreneurial } \\
\text { Leadership (Z) }\end{array}$} & Z1.1 & 0,634 & 0,402 & 0,598 & \multirow{5}{*}{0,791} \\
\hline & Z1.2 & 0,604 & 0,365 & 0,635 & \\
\hline & Z1.3 & 0,750 & 0,563 & 0,438 & \\
\hline & Z1.4 & 0,565 & 0,319 & 0,681 & \\
\hline & Z1.5 & 0,723 & 0,523 & 0,477 & \\
\hline
\end{tabular}

Note: All variables has construct reliability $\geq 0,70$, so the conclusion is reliable

\subsection{SEM assumption test}

a. Multivariate Normality

The multivariate $\mathrm{CR}$ is $-2,511$, the value is between -2.58 to +2.58 , so the multivariate data is normally distributed, or the assumption of multivariate normality is fulfilled.

b. Univariate Outlier

Z-score values on all indicators are in the range of -3 to +3 , so that the data univariately none are indicated as outliers.

c. Multivariate Outlier

\begin{tabular}{|c|c|c|c|}
\hline Observation number & Mahalanobis d-squared & $\mathrm{p} 1$ & $\mathrm{p} 2$ \\
\hline 187 & 46,931 &, 025 & ,994 \\
\hline 71 & 46,228 & 030 & ,982 \\
\hline 112 & 44,596 &, 042 & ,991 \\
\hline 160 & 44,248 & 045 & ,982 \\
\hline 132 & 43,586 &, 052 & ,980 \\
\hline 20 & 43,362 & 054 & 964 \\
\hline 51 & 42,511 &, 065 & ,977 \\
\hline 185 & 41,609 & 077 & ,989 \\
\hline 177 & 41,065 & 086 & ,991 \\
\hline 159 & 40,981 & 087 & ,983 \\
\hline 60 & 40,913 & ,088 & ,970 \\
\hline 49 & 39,699 &, 111 & ,995 \\
\hline 147 & 39,285 & ,119 & ,996 \\
\hline 6 & 38,554 & , 136 & ,999 \\
\hline 150 & 37,419 & , 165 & 1,000 \\
\hline 58 & 37,252 & 170 & 1,000 \\
\hline 69 & 37,220 & ,171 & 1,000 \\
\hline 89 & 37,205 &, 171 & 1,000 \\
\hline 130 & 37,191 & , 172 & ,999 \\
\hline 195 & 37,190 & , 172 & ,998 \\
\hline 26 & 29,789 & ,476 & 377 \\
\hline 126 & 29,775 & 477 & 332 \\
\hline 115 & 29,600 & ,486 & 375 \\
\hline
\end{tabular}


Chi-square table $=66.62$

The maximum value of the Mahalobis d-square is 46.931 , this value is still smaller than 66.62 , so there is no multivariate outlier in the research data, so all respondents can be used for analysis.

\subsection{SEM Model Test}

a. Base Model

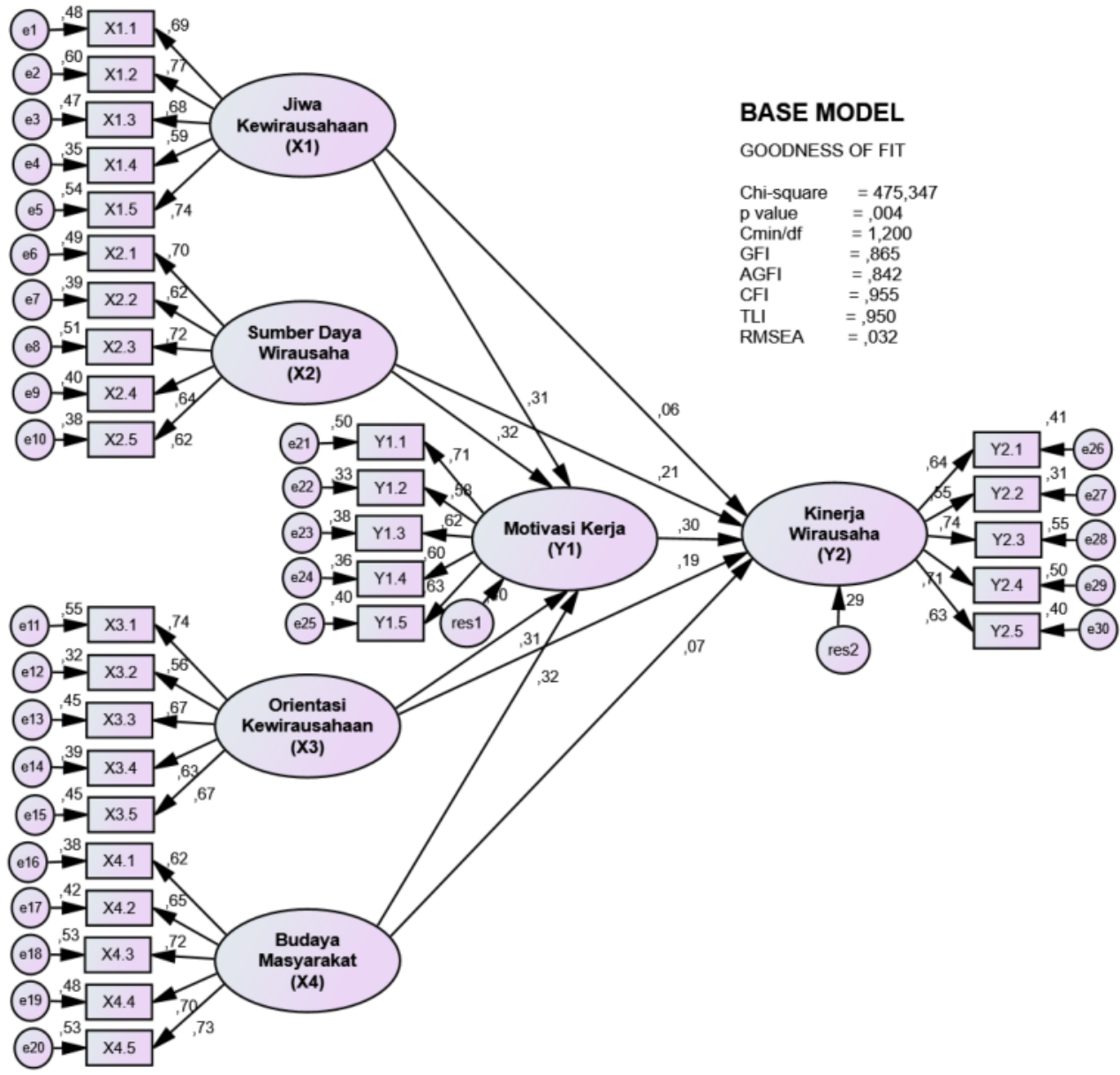

\begin{tabular}{|l|l|l|l|}
\hline $\begin{array}{l}\text { Criteria for } \\
\text { Goodness of Fit }\end{array}$ & Requirement & Test Result & Explanation \\
\hline P value chi-square & $\geq 5 \%$ & 0,004 & Tidak fit \\
\hline Cmin/df & $\leq 2,00$ & 1,200 & Fit \\
\hline RMSEA & $\leq 0,80$ & 0,032 & Fit \\
\hline GFI & $\geq 0,90$ & 0,865 & Marginal \\
\hline AGFI & $\geq 0,90$ & 0,842 & Marginal \\
\hline CFI & $\geq 0,95$ & 0,955 & Fit \\
\hline TLI & $\geq 0,95$ & 0,950 & Fit \\
\hline
\end{tabular}

Note: most of the goodness of fit criteria that meet the criteria (fit and marginal), so it can be concluded that the model is acceptable. But modification of the model needs to be done so that the model is more compatible with empirical data. Modification of the model is based on the modification values issued by Amos. 


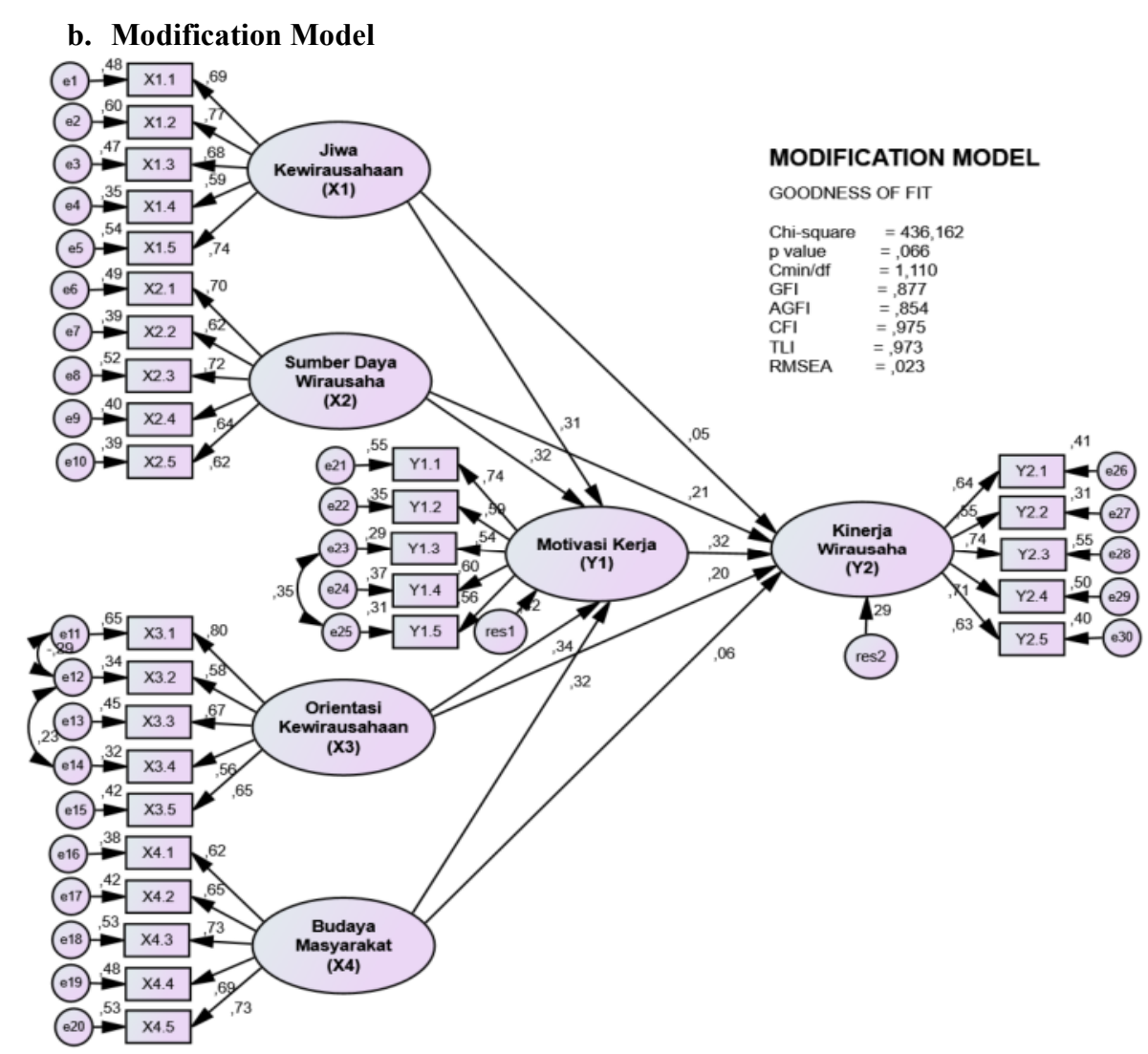

\begin{tabular}{|l|l|l|l|}
\hline $\begin{array}{l}\text { Criteria for } \\
\text { Goodness of Fit }\end{array}$ & Requirement & Test Result & Explanation \\
\hline P value chi-square & $\geq 5 \%$ & 0,066 & Fit \\
\hline Cmin/df & $\leq 2,00$ & 1,110 & Fit \\
\hline RMSEA & $\leq 0,80$ & 0,023 & Fit \\
\hline GFI & $\geq 0,90$ & 0,877 & Marginal \\
\hline AGFI & $\geq 0,90$ & 0,854 & Marginal \\
\hline CFI & $\geq 0,90$ & 0,975 & Fit \\
\hline TLI & $\geq 0,90$ & 0,973 & Fit \\
\hline
\end{tabular}

Note: all goodness of fit criteria meet the criteria, and have a far better compatibility than the initial model. Furthermore, it can be continued testing the influence between variables (hypothesis testing).

\subsection{Direct Influence Hypotheses Test Result}

\begin{tabular}{|c|ccc|c|c|c|c|}
\hline Hip. & \multicolumn{2}{|c|}{ Relationship between Variables } & Coef. & T count & $\begin{array}{c}P \\
\text { value }\end{array}$ & Explanation \\
\hline $\mathrm{H}_{1}$ & $\begin{array}{c}\text { Entrepreneurial } \\
\text { Spirit (X1) }\end{array} \rightarrow$ & $\begin{array}{c}\text { Work Motivation } \\
\text { (Y1) }\end{array}$ & 0,310 & 3,358 & 0,000 & Significant \\
\hline $\mathrm{H}_{2}$ & $\begin{array}{c}\text { Entrepreneurial } \\
\text { Resources (X2) }\end{array}$ & $\rightarrow$ & $\begin{array}{c}\text { Work Motivation } \\
\text { (Y1) }\end{array}$ & 0,319 & 3,481 & 0,000 & Significant \\
\hline $\mathrm{H}_{3}$ & $\begin{array}{c}\text { Entrepreneurial } \\
\text { Orientation (X3) }\end{array}$ & $\rightarrow$ & $\begin{array}{c}\text { Work Motivation } \\
\text { (Y1) }\end{array}$ & 0,341 & 3,818 & 0,000 & Significant \\
\hline $\mathrm{H}_{4}$ & $\begin{array}{c}\text { Community } \\
\text { Culture (X4) }\end{array}$ & $\rightarrow$ & $\begin{array}{c}\text { Work Motivation } \\
\text { (Y1) }\end{array}$ & 0,318 & 3,381 & 0,000 & Significant \\
\hline $\mathrm{H}_{5}$ & $\begin{array}{c}\text { Entrepreneurial } \\
\text { Spirit (X1) }\end{array}$ & $\rightarrow$ & $\begin{array}{c}\text { Entrepreneurial } \\
\text { Performance (Y2) }\end{array}$ & 0,050 & 0,555 & 0,579 & $\begin{array}{c}\text { Not } \\
\text { Significant }\end{array}$ \\
\hline $\mathrm{H}_{6}$ & $\begin{array}{c}\text { Entrepreneurial } \\
\text { Resources (X2) }\end{array}$ & $\rightarrow$ & $\begin{array}{c}\text { Entrepreneurial } \\
\text { Performance (Y2) }\end{array}$ & 0,206 & 2,170 & 0,030 & Significant \\
\hline
\end{tabular}




\begin{tabular}{|c|ccc|c|c|c|c|}
\hline $\mathrm{H}_{7}$ & $\begin{array}{c}\text { Entrepreneurial } \\
\text { Orientation (X3) }\end{array}$ & $\rightarrow$ & $\begin{array}{c}\text { Entrepreneurial } \\
\text { Performance (Y2) }\end{array}$ & 0,196 & 2,103 & 0,035 & Significant \\
\hline $\mathrm{H}_{8}$ & $\begin{array}{c}\text { Community } \\
\text { Culture (X4) }\end{array}$ & $\rightarrow$ & $\begin{array}{c}\text { Entrepreneurial } \\
\text { Performance (Y2) }\end{array}$ & 0,062 & 0,680 & 0,496 & $\begin{array}{c}\text { Not } \\
\text { Significant }\end{array}$ \\
\hline $\mathrm{H}_{9}$ & $\begin{array}{c}\text { Work Motivation } \\
\text { (Y1) }\end{array}$ & $\rightarrow$ & $\begin{array}{c}\text { Entrepreneurial } \\
\text { Performance (Y2) }\end{array}$ & 0,316 & 2,380 & 0,017 & Significant \\
\hline
\end{tabular}

\subsection{Hypothesis Testing Result of Moderation Effect}

The stages of testing the moderation effect (moderation effect) according to Baron and Kenny (1986) there are two stages. The first stage is to test the independent influence on the dependent must be significant, and the moderator influence on the dependent must also be significant. The second stage is testing the effect of interactions on the dependent must be significant, while the main influence that was previously significant, becomes insignificant. Stage 1 - Testing the Effect of Independent Variables and Moderator Variables on Dependent Variables

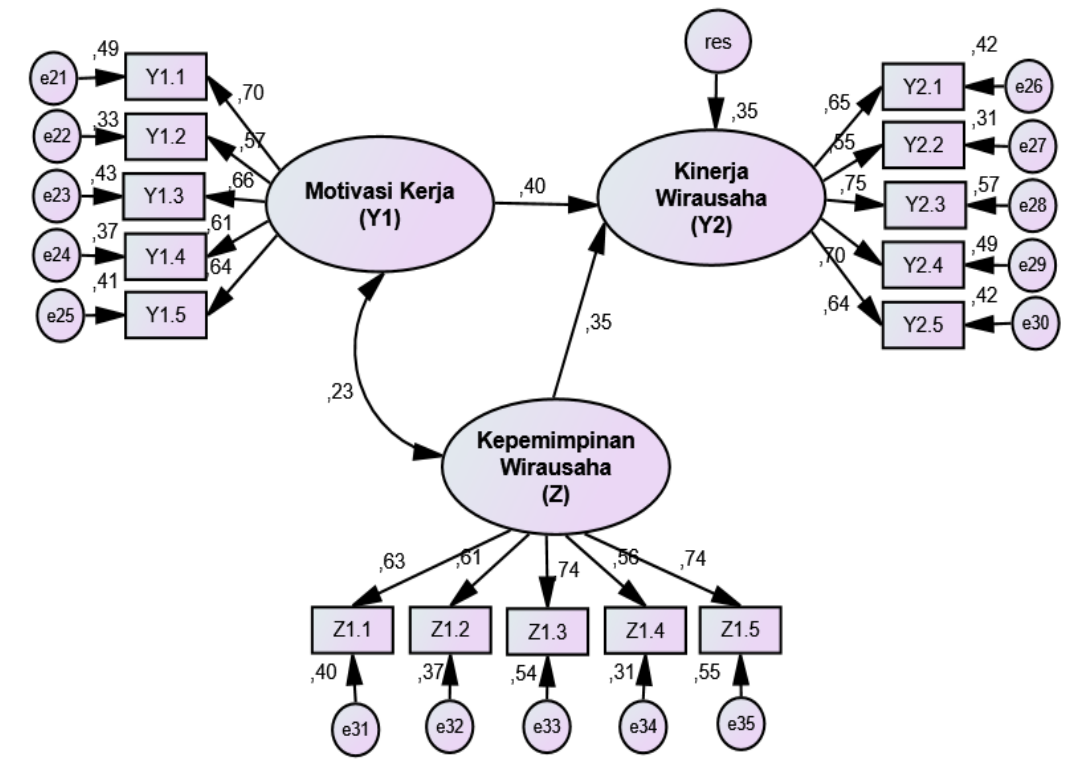

\begin{tabular}{|c|c|c|c|c|c|}
\hline \multicolumn{3}{|c|}{ Relationship between Variables } & Coef. & $\begin{array}{c}\mathrm{P} \\
\text { value }\end{array}$ & Exp. \\
\hline $\begin{array}{l}\text { Work Motivation } \\
\text { (Y1) }\end{array}$ & $\rightarrow$ & $\begin{array}{c}\text { Entrepreneurial } \\
\text { Performance (Y2) }\end{array}$ & 0,404 & 0,000 & Significant \\
\hline $\begin{array}{c}\text { Entrepreneurial } \\
\text { Leadership }(\mathrm{Z})\end{array}$ & $\rightarrow$ & $\begin{array}{c}\text { Entrepreneurial } \\
\text { Performance (Y2) }\end{array}$ & 0,347 & 0,000 & Significant \\
\hline
\end{tabular}

The influence of the independent variable on the dependent and the influence of the moderator variable on the dependent are both significant, so the testing of the effects of moderation can proceed to stage 2 
Stage 2 Test the Effect of Interaction on Dependent Variables

The moderating effect $\mathrm{Z}$ test model on the effect of $\mathrm{Y} 1$ on $\mathrm{Y} 2$ :

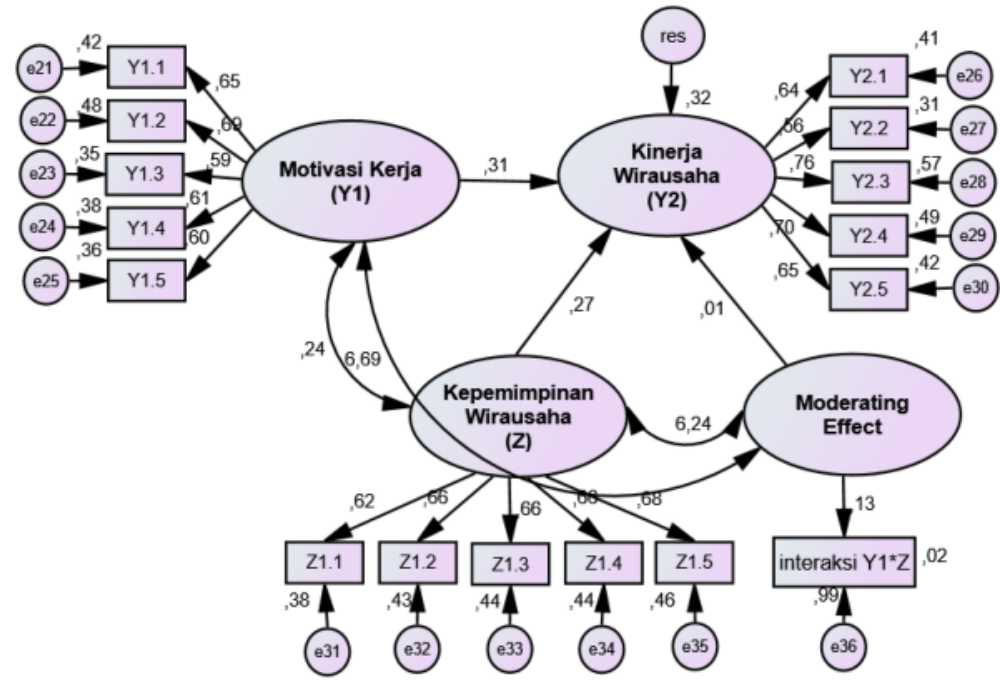

\begin{tabular}{|c|c|c|c|c|c|c|}
\hline Hip. & \multicolumn{3}{|c|}{ Relationship between Variables } & Coef. & $\begin{array}{c}\mathrm{P} \\
\text { value }\end{array}$ & Exp. \\
\hline \multirow{2}{*}{$\mathrm{H}_{10}$} & $\begin{array}{c}\text { Work } \\
\text { Motivation (Y1) }\end{array}$ & $\rightarrow$ & $\begin{array}{c}\text { Entrepreneurial } \\
\text { Performance (Y2) }\end{array}$ & 0,313 & 0,000 & Significant \\
\hline & $\begin{array}{c}\text { Interaction } \\
\mathrm{Z}^{*} \mathrm{Y} 1\end{array}$ & $\rightarrow$ & $\begin{array}{c}\text { Entrepreneurial } \\
\text { Performance (Y2) }\end{array}$ & 0,014 & 0,000 & Significant \\
\hline
\end{tabular}

Test of $\mathrm{H}_{10}$

The effect of interaction variables on OCB $(\mathrm{Z} * \mathrm{Y} 1 \rightarrow \mathrm{Y} 2)$ results in $\mathrm{p}$ value $<0.05$ so that the moderation is significant. The coefficient value of the interaction effect is 0.014 , this value is positive, meaning that entrepreneurial leadership is proven to strengthen the effect of work motivation on entrepreneurial performance, so that $\mathrm{H} 10$ is accepted. The effect of work motivation on entrepreneurial performance will be stronger in employees with good entrepreneurial leadership.

In testing the influence of moderation above there are two possibilities:

1. Pure Moderator $\rightarrow$ significant interaction effect and the main influence becomes insignificant.

2. Quasi Moderator $\rightarrow$ significant interaction effects and main influences remain significant

In testing the influence of moderation in this study, it is known that the influence of significant interactions and main effects remain significant, so that the moderating effects that occur are quasi moderators. This shows that entrepreneurial leadership in addition to being a moderator, can also be a predictor. Thus, entrepreneurial leadership in addition to being able to strengthen the effect of work motivation on entrepreneurial performance, is also a predictor that can directly affect entrepreneurial performance. 
Figure of All Hypotheses Test Result

\section{Explanation:}

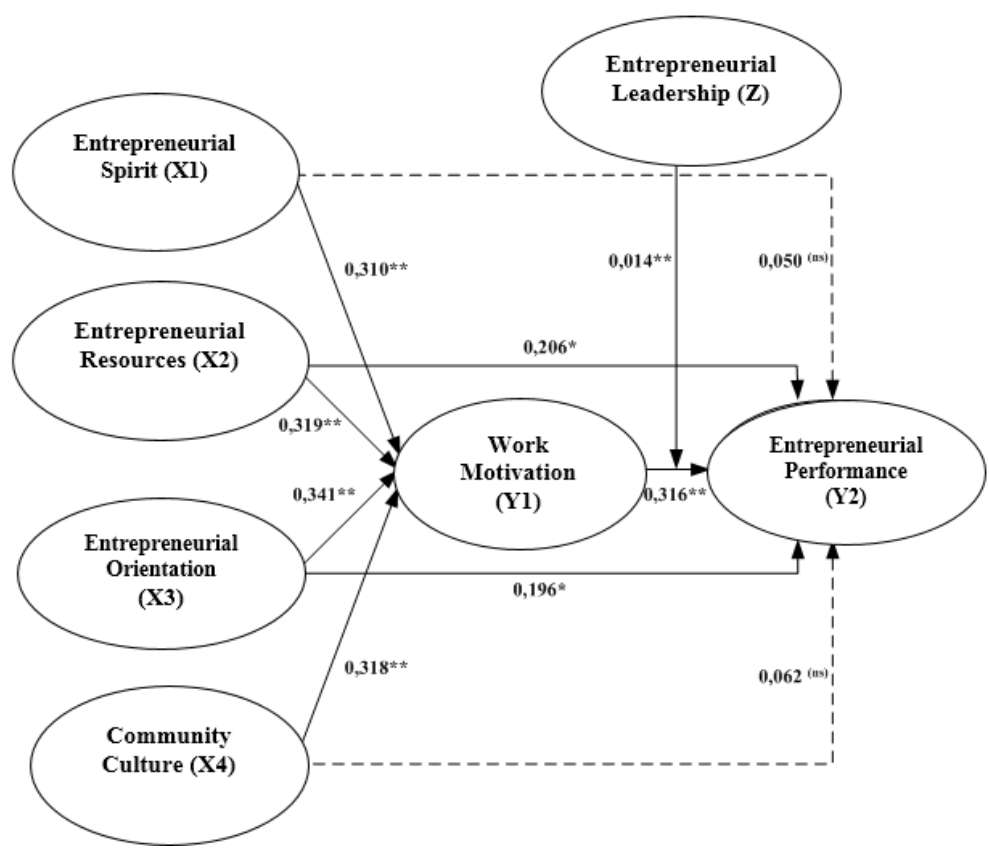

Dotted lines show insignificant effects

The multiple regression equation is as follows:

$\mathrm{Y} 1=0,310 \mathrm{X} 1+0,319 \mathrm{X} 2+0,341 \mathrm{X} 3+0,318 \mathrm{X} 4$

$\mathrm{Y} 2=0,050 \mathrm{X} 1+0,206 \mathrm{X} 2+0,196 \mathrm{X} 3+0,062 \mathrm{X} 4+0,316 \mathrm{Y} 1$

\section{Discussion}

From the data analysis the following research results were obtained:

1. The first hypothesis The entrepreneurial spirit affects the work motivation of the results of this study support the research conducted by Nikolic et al (2017), Pujiastuti (2013)

2. The second hypothesis is proven that entrepreneurial resources influence work motivation, the results of this study support research conducted by Haryani (2017), Abbas (2018)

3. The third hypothesis is proven that entrepreneurial orientation influences the motivation of this research work to support research that has been conducted by Purwanto and Trihudiyatmanto (2018), Marques et al (2012)

4. The fourth hypothesis is proven that community culture influences work motivation, the results of this study support the research conducted by Yuswani (2016), Ariffianti and Hamdi (2016)

5. The fifth hypothesis of this study turns out that entrepreneurial spirit does not affect Entrepreneurial Performance, this study does not support the research conducted by Hadijah (2019), Purwanti (2012)

6. The sixth hypothesis is proven that Entrepreneurial Resources influence Entrepreneurial Performance, the results of this study support the results of research conducted by Toyib (2017), Chein (2014)

7. The seventh hypothesis is proven entrepreneurial orientation influences entrepreneurial performance, the results of this study do not support research conducted by Purwanto and Trihudiyatmanto (2018), but support the research of Toyib (2017), Chein (2014)

8. The eighth hypothesis of Community Culture has no effect on entrepreneurial performance, the results of this study support the research conducted by Rante (2010), but do not support the research conducted by Yuswani (2016)

9. The ninth hypothesis Work motivation has an effect on proven entrepreneurial performance and this study supports the research conducted by Basuki (2007), Sumantri (2013)

10. The tenth hypothesis of Leadership is proven to moderate the effect of work motivation on performance, the results of this study support the research conducted by Hutagalung (2016) but do not support research conducted by Yulifah and Basiya (2015).

\section{Conclusion}

1. Entrepreneurial spirit, Entrepreneurial Resources, Entrepreneurial Orientation, Community culture influences 
work motivation is something that can be accepted logically because these four variables are unity factors that can form entrepreneurial work motivation. The existence of these four factors can strengthen efforts in the face of the harsh business climate in MSMEs in the community besides the toughness of an entrepreneur in running his business is needed because of the risks that must be faced in the future.

2. Entrepreneurial resources, entrepreneurial orientation affects the performance of entrepreneurs, this indicates that entrepreneurial resources have an important role in producing performance because of entrepreneurial capital as a source of business is needed. Besides that, the attitude of the entrepreneur in the orientation of his enthusiasm for work will support the maximum results in trying to fit into the occupied business. Motivation for personal encouragement to produce maximum performance is very necessary also because without the high motivation of course the work will not be obtained in accordance with the expectations of an entrepreneur.

3. The entrepreneurial spirit does not affect the performance of the entrepreneur. This can be explained that the entrepreneurial spirit of a businessman is already owned in accordance with his environment and the demands of his life and is embedded in each individual. Community culture does not affect the performance of entrepreneurs gives a picture that the culture of the community in which entrepreneurs are deeply embedded and are positive habits to conduct business activities for the sake of survival in the community. So weak or not the culture of the community then performance is a demand for an entrepreneur for the survival of his business.

4. Leadership factors make a significant contribution and are needed to strengthen motivation in creating entrepreneurial performance. An entrepreneurial leadership is needed to have good and broad insight in the future in order to maintain the continuity of the business, because future business conditions will be able to change without being planned in advance.

\section{Research Variables and Indicators}

Entrepreneurial spirit

1. Becoming an entrepreneur is an ideal starting from childhood

2. Being an entrepreneur helps the government to open up employment opportunities

3. Becoming entrepreneurs strengthens themselves to face life independently

4. Being an entrepreneur is challenged by the ever-changing business conditions

5. Becoming an entrepreneur is an alternative to achieving success without being dependent on others

Entrepreneurial Resources

1. Enough raw materials are available

2. Enough labor

3. Entrepreneurial funding loans are open from the government

4. The product market is still wide open

5. Have management ability to manage business ventures

Entrepreneurship Orientation

1. Doing business that is not dependent on others

2. Prioritizing productive activities

3. Anyone facing business risk

4. Challenge to surrender in the face of business competition

5. Doing business based on economic values

Community Culture of the Industrial Environment Society

1. The entrepreneurial environment supports the construction of business activities

2. The Islamic environment supports the production process

3. The attitudes and behavior of the surrounding community is oriented towards business ventures

4. The values of business success are believed with all my heart

5. Be honest in running a business

Work motivation

1. Always want to make changes for customer satisfaction

2. Oriented to useful actions

3. Enjoying businesses that provide challenges

4. Want to achieve success in life like other entrepreneurs

5. Preparing to face changes in the business climate that occurs at MSMEs

Entrepreneurial performance

1. Always try to produce a quality product 
2. Always maintain employee job satisfaction

3. Always maintain consumer satisfaction

4. Always try to do creativity and innovation on product development

5. Creating the cost of products and selling prices that are competitive.

Leadership

1. Able to manage time and activities as needed

2. Being able to see opportunities in the market

3. Maintain a harmonious relationship with employees

4. Manage business ventures based on abilities, skills and knowledge as well as experience.

5. Always optimistic in carrying out business activities

\section{References}

Abbas,Djamila.2018. Pengaruh Modal Usaha,Orientasi Pasar dan Orientasi Kewirausahaan Terhadap Kinerja UKM Kota Makasar. Jurnal Manajemen, Ide, Inspirasi (MINDS) ISSN-E:2597-6990; ISSN-P: $2442-$ 4951.Vol 5 No.1 hal. 95-111

Aramand,Majid. 2013. Women entrepreneurship in Mongolia: the role of culture on entrepreneurial motivation. Equality.Diversity and Inclusion: An International Journal.Vol.32 No.1 pp 68-82

Ardi,Fiko Aditya Delvi.2013. Pengaruh Lingkungan Kerja, Kompetensi dan Budaya lingkungan organisasi Terhadap Kinerja Pengrajin UMKM di Paguyuban Kampung Batik Kota Semarang. Tugas Akhir Fakultas Ekonomi Dan Bisnis Universitas Dian Nuswantoro Semarang

Arriffianti,Indah; Hamdi H.Nizar. Faktor-faktor yang mempengaruhi Motivasi Mahasiswa menjadi Wirausaha. Jurnal Valid Vo.13.No.1 Hal.1-10

Basuki.R (2007). Analisis hubungan antara Motivasi, Pengetahuan kewirausahaa, Kemandirian usaha terhadap Kinerja pengusaha pada karyawan industry kecil di daerah Pulogadung.Jurnal Usahawan 2(10) hal.1-8

Chein, Shih,Yi. 2014. Franchisor resources, spousal resources, entrepreuneurial orientation, and Performance in a copuple-owned franchise outlet. Management Decision Vo.52 No.5 pp.916-933 ISSN 0025-1747 DOI 10.1108/MD.07-2013-0368

Marques,Carla S.E; Ferriera,Joao J.M; Ferriera,Fernando A.F; Lages,Marrisa F.S. 2012 Entrepreneural orientation and Motivation to start up a Business Evidance from Helath Service Industry. Int.Entp.Manag.J P-ISSN 2622-8394 E-ISSN 2622-8122 Springer Science + Business Media Newyork.

Fauni,Arini Mayang.2017 Pengaruh Orientasi Kewirausahaan,Orientasi Pasar,Inovasi Usaha Dan Keunggulan bersaing TerhadapKinerja Usaha Mikro Kecil Dan Menengah Konveksi Kabupaten Kudus-Jawa Tengah.Tugas Akhir Program Pascasarjana. Program Studi Pendidikan Ekonomi Universitas Negeri Semarang..

Farmoni,Mariel; Arribas,Ivan; Vila,Jose E.2012. An Entrepreuner's social capital and performance The role of Access to Information in the Argentinean case. Discussion Paper in Economic Behaviour. Universidad Nacional de MAR DEL PLATA. FACULTAD de CIENCAS. ECONOMICAS SOCIALES.

Ferdinand, Augusty. 2014. Metode Penelitian Manajemen, Pedoman Penelitian Untuk Penulisan Skripsi, Tesis dan Desertasi Ilmu Manajemen. Penerbit BP Undip. Semarang.ISBN : 979-704-254-5

Gemina,Dwi; Silaningsih,Endang; Yuningsih,Erni.2016. Pengaruh Motivasi Usaha Terhadap Keberhasilan Usaha Dengan Kemampuan Usaha Sebagai Variabel Mediasi pada Industri Kecil Menengah Makanan Ringan Priangan Timur-Indonesia. Jurnal Manajemen Teknologi ISN 297-323 Vol.15 No.3

Hadijah,Siti.2019. Analisis Jiwa kewirausahaan dan Kreativitas terhadap Keberhasilan wirausaha Mahasiswa Universitas Mulawarman Angkatan 2014 (Studi pada Mahasiswa FKIP, FISIF, dan FEB). Ejournal Administrasi Bisnis ISSN.2355-5408. 7(1) 303-316

Haryani, Sri.2017. Pengaruh Lingkungan Kewirausahaan Terhadap Pengembangan Wirausaha Di Kabupaten Sleman. Ekuitas: Jurnal Ekonomi dan Keuangan. P-ISSN 2548-298X e-ISSN 2548-5024. Akreditasi No.80/Dikti/Kep/2012 DOI: 10.24034/j25485024.y2017.v1.i1.1841 hal. 24-43.

Hutagalung,Christofer.2016. Thesis : Efek moderator Gaya Kepemimpinan pada Pengaruh Motivasi Terhadap Kinerja Karyawan Kasus pada Karyawan Tetap Dinas Perhubungan dan Transpotasi Daerah Yogyakarta. Fakultas Ekonomi Universitas Sanata Darma Yogyakarta.

Kasali,Rhenald; Nasution,Arman Hakin; Purnomo,Boyke; $\quad$ Ciptarahayu,Agustina; Mirzanti,IstiRaafaldinini;Rustiadi,Sonny;Daryanto,Heni Kuswanti;Mulyana,Asep.2010.Modil Kewirausahaan,Hikmah (PT.Mizan Publika).Jakarta ISBN 978-979-3714-99-8

Machmud,Senen; Sidharta,Iwan. Entrepreneural Motivation and Business Performance of SMEs in the Suci Clothing Center, Bandung, Indonesia. DLSU Business \& Economics Review Vol.25 No.2 pp 63-78. JEL Classifications :E24,J24, L26. De La Salle University 
Nicolik,Milan; Cockalo,drgan; Terek,Edit; Bozic,Sanja; ; Nastasic,Aleksandra.2017. The Impact of Life Values on Intrepreneurial Intentions of Studies in Serbia. Journal of Engineering Management and Competitivness (JEMC) ISSN.2217-8147 (online) Vol.7 No.1 pp.28-34

Nitisusastro,Mulyadi.2009. Kewirausahaan Dan Manajemen Usaha Kecil. Penerbit Alfabeta.BandungISBN 978602-8361-68-2

Nouthouse,Peter G. 2013, Kepemimpinan.Teori dan Praktitk. Penerbit Indeks. Jakarta. ISBN 979-062-434-4

Pujiastuti,Eny Endah.2013 Peran Penting Jiwa Kewirausahaan Dalam Pengembangan Usaha Baru Pasca Bencana. Proceeding Konferensi Nasional II Media Bis nis dan Perdamaian hal.23-44 Fisip UPN "Veteran" Yogyakarta.

Purwanti,Endang.2012. Pengaruh Karakteristik Wirausaha,Modal Usaha,Strtegi Pemasaran Terhadap Perkembangan UMKM Di Desa Dayaan Dan Kalilondo Salatiga. Among Makarti.Vol.5 No.9

Purwanto,Heri; Trihudiyatmanto.M.2018. Pengaruh Intensi Berwirausaha, Oreintasi Kewirausahaan Terhadap Kinerja Usaha Dengan Motivasi sebagai variabel intervening pada Sentra UMKM Carica di Wonosobo Jawa Tengah. Journal of economic,Management,Accounting and Technology Vol 1 No 1 hal. 42-52

Rante,Yohanes.2010. Pengaruh Budaya Etnis dan Perilaku Kewirausahaan Terhadap Kinerja Usaha Mikro Kecil Agribisnis di Provinsi Papua. Jurnal Manajemen Dan Kewirausahaan Vo.12.No.2

Robbins,Stephen P.;Judge,Timothy A. 2015 Perilaku Organisasi. Penerbit Salemba Empat. ISBN 978-979-691460-9

Subanar,Harimurti.2012. Manajemen Usaha Kecil. BPFE, Yogyakarta. Fakultas Ekonomika Dan Bisnis UGM ISBN 979-503-344-1

Sugiyono. 2010. Metode Penelitian Kuantitatif, Kualitatif dan R\&D. Penerbit Alfabeta.Bandung.

Saiman,Leonardus.2014. Kewirausahaan. Teori,Praktik dan Kasus-kasus. Penerbit Salemab Empat ISBN 978979-061-450-5

Sumantri,Bayu (2013) Pengaruh Jiwa Kewirausahaan Terhadap Kinerja Usaha Wirausaha Wanita Pada Industri Pangan Rumahan di Bogor.Thesis Sekolah Pascasarjana Institut Pertanian Bogor, Program Studi Agribisnis.

Suryana.2013.Kewirausahaan. Kiat Dan Proses Menuju Sukses. Penerbit Salemba Empat Jakarta ISBN 978-979061-375-1

Toyib,Jein Sriana.2017. Pengaruh Sumberdaya dan orientasi Kewirausahaan Terhadap Kinerja Usaha Kecil Dan Menengah. Fakultas Ekonomi Dan Bisnis Universitas Papua. eMa Jurnal Manajemen Vol.12 No.2 Hal. $1-18$

Purwanto,Heri Trihudiyatmanto,M.2018. Pengaruh Intensi Berwirausaha, Orentasi Kewirausahaan Terhadap Kinerja Usaha dengan Motivasi sebagai Variabel Intervening Pada Sentra UMKM Cariga Di Wonosobo. Thesis Universitas Sains Al-Qur'an (UNSIQ) hal. 1-13

Widiyatnoto,Erfikas.2013. Pengaruh Jiwa Kewirausahaan dan Budaya Ke;uarga Terhadap minat berwirausaha pada Siswa SMKN 1 dan SMKN 2 Wonosari di Kabupaten Gunungkidul Jawa tengah

Yulifah,Lafi; Basiya,Robertus.2015. Pricipal Leadership Moderates Motivation and Work Discipline Effects on Teacher Performance. Jurnal Mahasiswa https://www.unisbank.ac.id/ojs/index.php/pasca1/artice;/view/3599

Yuswani,wiwik.2016 pengaruh Budaya dan stress Terhadap Motivasi Kerja dan dampaknya terhadap kinerja karyawan pada PT.Kerinci Permata Motor Jambi. Jurnal Sinstech Politeknik Indonusa Surakarta ISSN : 2355-5009 Vol 2 No.5 Hal.1-10 